\title{
DETERMINACIÓN DE LA EDAD DE APROVECHAMIENTO DE INDIVIDUOS DE Prosopis alba MEDIANTE UN MODELO LOGISTICO DE INTERCEPTO ALEATORIO PARA INCREMENTOS RADIALES
}

Juárez de Galindez, M "; Giménez, A. M 2; Rios N 3 ; Balzarini, M $^{4}$

\section{RESUMEN}

De las 15 especies arbóreas nativas del Parque Chaqueño semiárido en Argentina, el algarrobo blanco (Prosopis alba Griseb) pertenece a la categoria de especies secundarias con alta variabilidad de patrones de crecimiento entre individuos. Es una de las mimosáceas argentinas de mayor importancia económica, ya que la madera, es muy cotizada para muebles, carpinteria de obra, pisos y revestimientos. Su creciente utilización también para leña, forraje y alimentos hace que el recurso sea muy explotado. Según los principios básicos de un aprovechamiento racional y sostenido, no se debe aprovechar los bosques a una tasa mayor que la de su crecimiento. Por ello es necesario estimar el patrón de crecimiento biológico para que los árboles no sean utilizados antes o después de la edad de culminación del crecimiento biológico.

El objetivo de este trabajo es determinar la edad de aprovechamiento de un individuo "tipo" de algarrobo blanco (Prosopis alba) que crece en Santiago del Estero, Argentina, bajo condiciones no limitantes más allá de las asociadas a caracteristicas climáticas propias de la región. Para ello, se filtró variaciones debidas al clima regional y otros disturbios y se modeló la señal biológica contenida en las series de anchos de anillos con un modelo logistico de intercepto aleatorio para contemplar y entender mejor variaciones del crecimiento entre individuos de la misma especie bajo las mismas condiciones ambientales. La esperanza marginal del modelo logistico mixto fue usada para construir curvas de incremento corriente anual y medio que, superpuestas en un mismo plano temporal, permitieron identificar la edad a la cual estos crecimientos son equivalentes (edad de culminación biológica). Se encontró que, para un árbol tipo de algarrobo blanco creciendo en esta región del Chaco Semiárido, el crecimiento anual promedio es de $0.40 \mathrm{~cm} / a n ̃ o$ y que el comienzo en la disminución del mismo podria esperarse alrededor de los 60 años. A pesar del descenso en la tasa de crecimiento a partir de los 60 años y debido a la acumulación de masa maderable, el estudio sugiere que no es conveniente que el aprovechamiento de estos árboles se realice antes de los 90 años de edad.

Palabras clave: Algarrobo blanco, crecimiento, incrementos radiales, modelo logistico

\footnotetext{
'Cátedra de Estadistica

${ }^{2}$ Cátedra de Dendrologia

${ }^{3}$ Cátedra de Dasometría. Universidad Nacional de Santiago del Estero. Facultad de Ciencias Forestales.-Instituto de Silvicultura y Manejo de Bosques. Santiago del Estero. Argentina. Tel/Fax (0385) 450-9550. E-mail: marga@unse edu.ar ${ }^{4}$ Estadistica y Biometría, CONICET- Fac. de Ciencias Agropecuarias, Universidad Nacional de Córdoba
} 


\section{ABSTRACT}

Out of the 15 native tree species in the Semiarid Chaco Park, the algarrobo blanco (Prosopis alba Griseb.) belongs to the so-called secondary species category showing high interindividual variability in growth patterns. From an economical standpoint, it is one of the most important Argentinean mimosae since its wood is highly valued for the making of furniture, builtin carpentry, parquet, and coverings. It is increasingly utilized as charcoal, forage and food as well which altogether make it an extensively exploited resource. In accordance with the basic principles for a rational and sustained harvesting, forests must not be exploited at a rate that is higher than that of its development. Thus, the biological growth pattern needs to be estimated so that trees are not harvested before or after the age their biological growth is reached.

The aim of this paper is to determine the age at which an average individual of algarrobo blanco (Prosopis alba) growing in Santiago del Estero. Argentina, is better harvested under no other limiting conditions than those associated to the climate of the region. Thus, using a logistic model for random interception and keeping variations owed to climate and other disturbances apart, the biological signal contained in the series of ring widths was modeled for a better observation and understanding of variations among individuals of the same species and under the same conditions. Using the marginal hope of the mixed logistic model both the annual and average growth curves were plotted and the age at which they are equivalent (i.e. biological culminating age) was identified by superposing them at a same temporal plane. It was found that $0.40 \mathrm{~cm}$ per year is the average annual growth for a typical individual of algarrobo blanco growing in this region of the Semiarid Chaco Park and that its growth decrease might be expected to occur when it is about 60 years old. Despite the latter and due to the timberable mass, this study suggests that these trees should not be harvested before they are 90 years old.

Key words: Algarrobo blanco, growth, radial increment, logistic model. 


\section{INTRODUCCIÓN}

En los últimos 65 años la reducción de bosques y montes naturales en Santiago del Estero, Argentina, ha sido del orden del $69 \%$ de la masa forestal inicial, desmontándose en promedio alrededor de $11395 \mathrm{~km}^{2} /$ año (Naumann y Madariaga, 2003). Para revertir esta situación, es necesario tomar medidas que deberán basarse necesariamente en investigaciones sobre la biologia y productividad de los bosques (Giménez, 1998). Los estudios de crecimiento son importantes para los encargados de planificar el manejo y la conservación forestal. De las 15 especies arbóreas de Santiago del Estero, el algarrobo blanco (Prosopis alba Griseb) pertenece a la categoria de especies secundarias, por formar parte del estrato intermedio con ejemplares de 8 a 12 metros de altura. Es una de las mimosáceas argentinas de mayor importancia económica (Giménez et al., 1998).

Su madera, es muy cotizada para muebles, carpinteria de obra, parquet, revestimientos. Su creciente utilización también para leña, forraje y alimentos hace que este recurso sea explotado sin tener en cuenta los principios básicos de un aprovechamiento racional y sostenido; por ello existe interés en determinar la edad de aprovechamiento más precisamente y para lo cual es necesario modelar el crecimiento. La modelación del crecimiento del leño de especies nativas en su ambiente permite la estimación de los momentos donde los árboles alcanzan el máximo crecimiento medio, información de utilidad para una apropiada corta, es decir una corta que se realice en un momento en el que se asegura la renovación (Perpiñal et al., 1995).

El modelado estadistico del crecimiento biológico forestal se realiza más frecuentemente en términos de rodales coetáneos. Cuando éstos no son coetáneos es necesario realizar un pre-tratamiento de las series de anchos de anillos de crecimiento para identificar la señal que se pretende modelar (señal debida a la edad del árbol). Eliminadas las variaciones debida al clima o disturbios de sitio para cada serie de ancho de anillos, generalmente se modela una curva de crecimiento promedio poblacional que representa el promedio de los crecimientos logrados por un número de árboles para una misma edad de su ciclo de vida.

Distintos modelos lineales (Graybill, 1976) y no lineales (Lee, 1982) en sus parámetros son utilizados en la modelación de tendencia de curvas de crecimiento biológico poblacional. En Prosopis alba Griseb, Juárez de Galindez et al (2005) comparan los modelos no lineales de Chapman-Richards y logistico sobre series dendrocronológicas previamente suavizadas para descontar la variabilidad debida a variaciones climáticas, encontrando que el modelo logistico provee un mejor ajuste que el modelo de Chapman-Richard y que cualquier modelo lineal. La función logistica es frecuentemente utilizada como modelo para la explicación de variaciones de incrementos de anchos de anillos en las ciencias forestales, en función de la edad biológica. Más allá de la aplicabilidad de este modelo no lineal a nivel poblacional, numerosos estudios de crecimiento con especies nativas del Chaco Semiárido sugieren una gran variabilidad entre árboles de la misma especie y por tanto las curvas promedios poblacionales pueden ser cuestionables.

Las diferencias en patrones de crecimiento de individuo a individuo en Prosopis alba en el Chaco Semiárido Argentino son mayores a las que se podrian modelar estadisticamente con un modelo de regresión que contenga sólo un término aleatorio de error. Mediante el enfoque de los modelos mixtos, es posible obtener un modelo que capte la estructura general 
contemplando la variabilidad tanto inter como intra-árbol (Balzarini et al, 2004).

Nuevas aplicaciones de modelos no lineales mixtos con uno o más coeficientes de regresión aleatorios (Molenbergh y Verbeke, 2007) han mostrado ser apropiadas para modelar estos crecimientos hipervariables. La interpretación de los parámetros del modelo con coeficientes aleatorios depende de la naturaleza lineal o no lineal de la función usada para expresar el crecimiento. Los modelos de crecimiento del tipo mixtos no lineales, como podria ser un modelo logistico con coeficientes aleatorios, permiten inferir tanto sobre crecimientos de árboles especificos como sobre patrones típicos o caracteristicos de una población de árboles.

En los últimos años, en la República Argentina, se realizó estudios sobre el crecimiento en especies leñosas nativas: Perpiñal et al (1995); Giménez (1998); Giménez et al. (1998); Gimenez et al., (2000); Juárez de Galindez (2001); Juárez de Galindez et al (2003); Juárez de Galindez y Balzarini (2003); Juárez de Galindez et al (2005); Juárez de Galindez et al (2007)a; Juárez de Galindez et al. (2007)b. En ellos se abordó las curvas de crecimiento bajo modelos de efectos fijos lineales y no lineales y también bajo modelos sujeto-especifico en el marco teórico de los modelos lineales mixtos pero no se trabajó con modelos mixtos no lineales.

El objetivo de este trabajo es modelar el crecimiento poblacional en diámetro de algarrobo blanco (Prosopis alba) en el Chaco Semiárido de Argentina, con un modelo logístico que incorpore un componente aleatorio a fin de contemplar mejor las variaciones de crecimiento entre individuos. La finalidad además es la aplicación del modelo ajustado en la determinación e interpretación de la edad óptima de aprovechamiento de individuos típicos de algarrobo blanco en el área de interés.

\section{MATERIAL Y MÉTODOS}

El estudio se realizó con árboles provenientes de distintas localidades de Santiago del Estero, provincia argentina que forma parte de la Región del Chaco Semiárido. El clima de la provincia es cálido, con temperaturas bastante uniformes, cuyas máximas absolutas superan los $45^{\circ} \mathrm{C}$ en verano y temperaturas mínimas absolutas de hasta $-10^{\circ} \mathrm{C}$ en invierno. Las precipitaciones oscilan de $750 \mathrm{~mm}$ en la parte oriental a $500 \mathrm{~mm}$ al oeste del territorio provincial, disminuyendo progresivamente de este a oeste siendo el balance hídrico deficitario en la mayor parte de la provincia, en todos los meses del año (Boletta, 1988).

De una muestra de árboles de algarrobo blanco de distintos tamaños, tomada al azar dentro de rodales de distintas zonas de la provincia, se escogió aleatoriamente 10 individuos de aquellos que cumplian con las siguientes caracteristicas: adultos (diámetro a la altura del pecho superior a $30 \mathrm{~cm}$, con corteza); calidad del fuste (sin defectos); posición social (árbol dominante); tipo de copa (simétrica) y vitalidad (sano) para evitar modelar el crecimiento de árboles con fuertes limitaciones o disturbios no comunes. Los individuos seleccionados fueron apeados durante 1997 en las localidades Loreto (LS. 28¹8'3.74", LO. 64¹0'44.95"); Las Delicias, (LS.

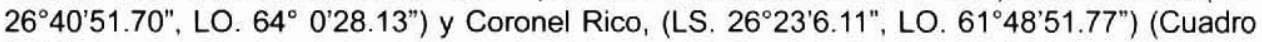
$\left.\mathrm{N}^{\circ} 1\right)$. 


\section{Cuadro $\mathrm{N}^{\circ} 1$}

\section{DIÁMETRO A LA ALTURA DEL PECHO, ALTURA Y EDAD DE LOS INDIVIDUOS DE ALGARROBO BLANCO ESTUDIADOS ORDENADOS POR LUGAR DE ORIGEN}

\begin{tabular}{|c|c|r|r|c|}
\hline Árbol & DAP (cm) & \multicolumn{1}{|c|}{$\begin{array}{c}\text { Altura Total } \\
(\mathbf{m})\end{array}$} & $\begin{array}{c}\text { Edad } \\
\text { (afios) }\end{array}$ & Zona \\
\hline 1 & 57,29 & 7,86 & 107 & Loreto \\
\hline 2 & 30,83 & 10,13 & 47 & Loreto \\
\hline 3 & 53,65 & 9,80 & 61 & Las Delicias \\
\hline 4 & 51,56 & 7,63 & 85 & Las Delicias \\
\hline 5 & 31,64 & 8,31 & 55 & Las Delicias \\
\hline 6 & 60,78 & 12,00 & 113 & Las Delicias \\
\hline 7 & 45,11 & 8,78 & 37 & Coronel Rico \\
\hline 8 & 44,40 & 9,62 & 61 & Coronel Rico \\
\hline 9 & 45,77 & 12,89 & 67 & Coronel Rico \\
\hline 10 & 87,03 & 14,04 & 95 & Coronel Rico \\
\hline
\end{tabular}

De los árboles seleccionados se extrajo tortas de $5 \mathrm{~cm}$ de espesor a la altura de 0.30 m (base). Las muestras fueron preparadas en la forma descrita por Giménez et al. en 1998; mediante el equipo computarizado ANIOL y el programa CATRAS (Aniol, 1991) se realizó la marcación y medición del espesor de anillos de crecimiento (Giménez, 1998; Moglia, 1999). Los diámetros para cada edad, fueron obtenidos mediante análisis epidométrico del fuste realizado sobre cada individuo (Rios et al., 1997). El programa de análisis epidométrico de fuste (Ríos et al., 1996) fue usado para promediar el espesor de los anillos medidos en cuatro orientaciones (radios) y con ellos obtener una serie de espesores de anillos en cada edad para cada uno de los individuos estudiados.

Suponiendo que el crecimiento de un árbol, en un periodo de tiempo determinado, es el resultado de la influencia de distintos factores: edad del árbol, clima y disturbios (Villalba, 1988, Catalán, 2000), algunos autores (Perpiñal et al., 1995; Juárez de Galíndez, 2001; Juárez de Galindez et al., 2003), al trabajar con especies nativas, usan la técnica de suavizado de las series individuales. El suavizado o filtrado de las series dendrocronológicas permite eliminar la variación debida al clima $u$ otros disturbios que afectan cada árbol a una edad particular antes de la modelación del crecimiento biológico poblacional. Asi la técnica de suavizado es interpretada como una herramienta para separar la señal de interés (señal debida a la edad del árbol) del ruido proveniente de otras fuentes de variación que afectan los anchos de los anillos de crecimiento de un árbol.

Para suavizar las series de anchos de anillo, fue empleado un filtro basado en medias móviles de tamaño de ventana igual a 7 años (Cleveland, 1979) implementado en el software estadístico InfoStat (InfoStat, 2008). Este filtro de baja frecuencia permitió rescatar la tendencia a largo plazo, la cual se supone refleja aquélla debida a la edad del árbol o al crecimiento biológico. En la Figura $\mathrm{N}^{\circ} 1$ se muestra las series de anchos de anillos directamente como fueron observadas y luego del suavizado para cada uno de los árboles que participaron del modelado. 

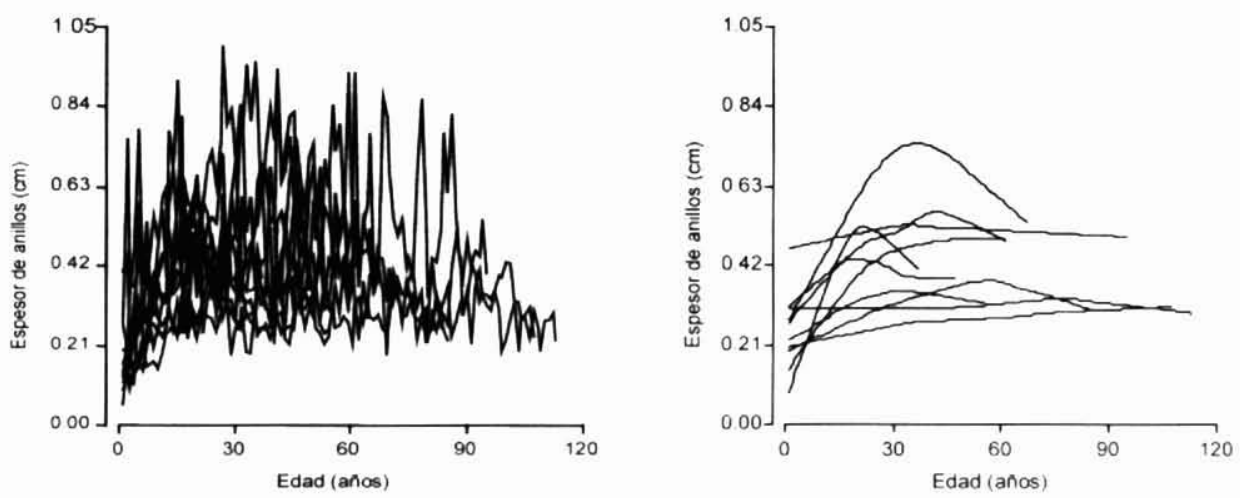

Figura $\mathbf{N}^{\circ} 1$

INCREMENTOS RADIALES OBSERVADOS (IZQUIERDA) Y SUAVIZADOS (DERECHA) DE 10 ÁRBOLES DE ALGARROBO BLANCO EN EL CHACO SEMIÁRIDO ARGENTINO

El número de mediciones de ancho de anillos para cada árbol varia pues depende de la edad que, como se muestra en el Cuadro $\mathrm{N}^{\circ} 1$, fue altamente variable. Por otra parte, las mediciones de anchos de anillos desde una muestra dendrocronológica constituyen medidas repetidas en el mismo individuo que se caracterizan por estar serialmente correlacionadas. Las correlaciones seriales pueden tratarse de manera explícita o implícitamente mediante la incorporación en el modelo de un termino aleatorio distinto al término de error clásico que asocie las mediciones repetidas sobre un mismo individuo (Balzarini et al, 2004). Es decir se espera la presencia de un efecto de individuo o serie dendrocronológica que agrupe el conjunto de mediciones de una misma serie. Además de las correlaciones seriales esperadas por la longitudinalidad del fenómeno de crecimiento biológico sobre un individuo, se esperan correlaciones entre las mediciones de una misma serie generadas por el mismo proceso de suavizado o filtrado del pre-tratamiento de la serie. Para la estructura de media, debido a los antecedentes de modelado de crecimiento en Prosopis, se seleccionó un modelo logistico. La forma integrada (función de rendimiento) del modelo logístico que expresa el incremento radial esperado a una cierta edad es:

$$
y_{j}=\frac{b_{0}}{1+b_{1} * e^{h_{2}^{x j}}}
$$

Donde:

$y_{\text {il }}$ es el incremento radial del individuo i-ésimo en el tiempo j infinito

$b_{0}$ es la asíntota, es decir el valor de la función cuando $x$ (edad del árbol) tiende a 
b está relacionado con la ordenada al origen

$\mathrm{b}$, es un parámetro tasa relacionado con el valor de $x$ (edad del árbol en el tiempo j).

Lindstrom y Bates (1990) modelan el crecimiento en circunferencia de árboles bajo el modelo logístico pero toman al intercepto como aleatorio ya que éste parecia ser realmente el único parámetro que variaba de un árbol a otro produciendo una familia de curvas logisticas con corrimiento en la ordenada al origen que reflejaban adecuadamente la variación interindividual. El incremento radial esperado en el modelo logístico mixto, con intercepto aleatorio, para el i-ésimo individuo, se puede expresar como:

$$
y_{i j}=\frac{b_{0}+u_{i}}{1+b_{1} * e^{x j}}
$$

Generalmente se asume que tanto los errores $\left(\varepsilon_{\|}\right)$asociados a cada observación como las componentes aleatorias que reflejan el efecto individuo $(u)$ son independientes y se distribuyen como variables aleatorias normales de media cero y varianzas constantes $\left(\sigma^{2}\right.$ denota la varianza residual y $\sigma_{u}^{2}$ la componente de varianza asociada a la variación entre los efectos $u$ ).

La derivada de la función de rendimiento, también conocida como curva de crecimiento esperada para el individuo i-ésimo, en el contexto del modelo mixto, puede entonces expresarse como:

$$
y_{i}=\frac{b_{11}+u_{i}}{\left(1+b_{1} * \exp \left(-x / b_{2}\right)^{2}\right.} * \frac{b_{1}}{b_{2}} * \exp \left(-x / b_{2}\right)
$$

La estimación de este modelo por métodos basados en la verosimilitud, demanda integrar la expresión del modelo a través de la distribución de los efectos aleatorios. Los algoritmos computacionales usados en la estimación incluyen no solo aspectos de optimización sino también de integración. Para ajustar este tipo de modelos No Lineales en SAS (versión 9.1) se usó Proc NLMIXED (Littell et al., 1996) para el que es necesario especificar valores iniciales de los parámetros. Este procedimiento ajusta el modelo mixto no lineal maximizando una aproximación a la probabilidad integrada sobre los efectos aleatorios (Wolfinger, 1993). NLMIXED permite usar datos con distintas distribuciones para la variable respuesta y efectos aleatorios normales. El método de integración usado fue la adaptativa Gaussiana y la técnica de optimización conocida como Dual Quasi-Newton con un solo punto de cuadratura. Los puntos de cuadratura no están alrededor de cero sino que se seleccionan en una región apropiada del integrando (Machiavelli, 2007).

Como medidas de bondad de ajuste se usó: EAM (media de los errores absolutos), Errores estándares relativos de los estimadores (error estándar dividido por la estimación del parámetro) y gráfico de residuos estudentizados vs valores predichos. Los residuos estudentizados son 
interpretados como los residuos comunes (diferencia entre el valor observado y el predicho) divididos por una función que no sólo incluye la variabilidad total sino también la influencia del dato en el ajuste, dependiente de la edad del árbol en la que se registra el crecimiento ya que las edades extremas (bajas o altas) pueden ser muy influyentes en el mismo. El modelo ajustado fue usado para determinar la edad de aprovechamiento recomendada según la intersección de la curva de incremento radial corriente anual con la curva de incremento radial medio anual que sugiere el ajuste (Perpiñal et al., 1995).

\section{RESULTADOS}

Los parámetros del modelo marginal resultante son incluidos en el Cuadro $\mathrm{N}^{\circ} 2$. La tasa de crecimiento expresada como el cociente entre la edad del árbol y $\mathrm{b}_{2}$ es igual a 0,023 . Los predictores de los efectos aleatorios que permiten describir variaciones entre árboles pueden ser observados en el Cuadro $\mathrm{N}^{\circ} 3$, junto a las predicciones del incremento corriente anual que se espera para cada individuo a los 55 años y a los 85 años. La desviación estándar de los efectos aleatorios, que puede ser interpretada como una medida de la covarianza entre los datos del mismo árbol, es de 18,9, la cual resultó ser estadisticamente significativa según el contraste sugerido por Molenbergh y Verbeke (2007) para este tipo de modelos. El desvio estándar de los términos de error fue solo 0,048 .

\section{Cuadro $\mathrm{N}^{\circ} 2$ \\ PARÁMETROS DEL MODELO LOGISTICO MARGINAL. CURVA DE CRECIMIENTO}

\begin{tabular}{|c|r|r|c|}
\hline Parámetro & Estimación & \multicolumn{1}{|c|}{$\begin{array}{c}\text { Error } \\
\text { estándar }\end{array}$} & $\begin{array}{c}\text { Error } \\
\text { estándar } \\
\text { relativo }(\%)^{\star}\end{array}$ \\
\hline $\mathbf{b}_{0}$ & 78,8533 & 6,3164 & 8 \\
\hline $\mathbf{b}_{1}$ & 3,7831 & 0,1063 & 3 \\
\hline $\mathbf{b}_{2}$ & 43,2454 & 1,1901 & 3 \\
\hline
\end{tabular}

- Porcentaje del error estándar respecto a la estimación del parámetro 


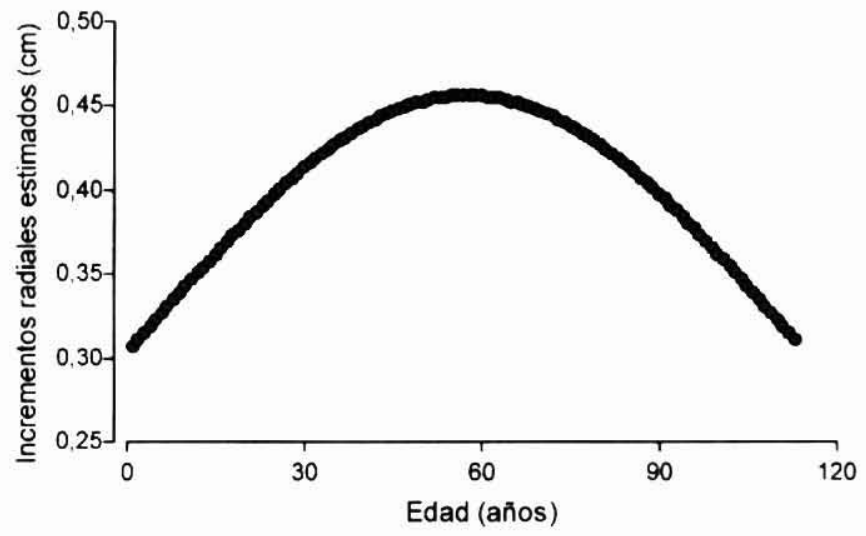

Figura $\mathrm{N}^{\circ} 2$

INCREMENTOS RADIALES ESTIMADOS EN FUNCIÓN DE LA EDAD DEL ÁRBOL

Se observa en la Figura $N^{\circ} 2$ que la disminución del crecimiento comienza poco antes de los 60 años.

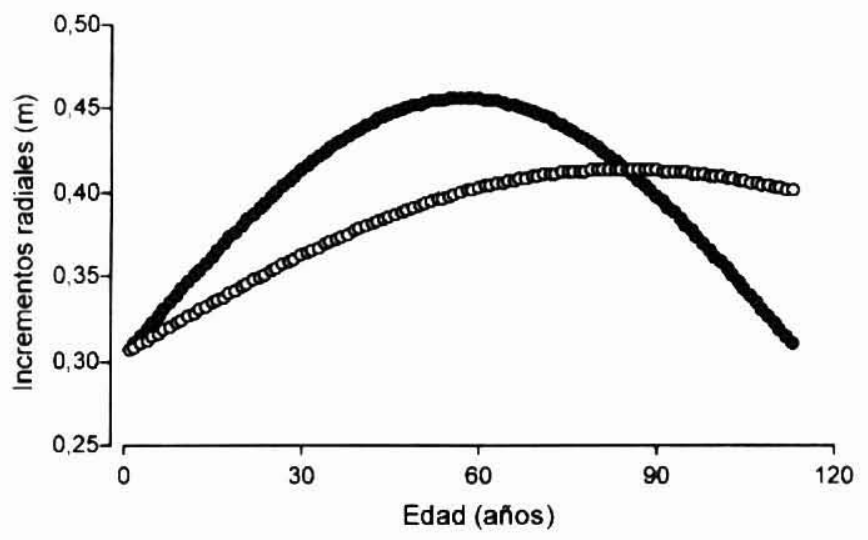

Figura $\mathrm{N}^{\circ} 3$

PUNTO DE CULMINACIÓN DEL CRECIMIENTO BIOLÓGICO

El punto de corte de las curvas de incremento corriente anual (o de crecimiento) y la curva de incremento medio anual se encuentra próximo a los 90 años (Figura $\mathrm{N}^{\circ} 3$ ). A los 85 años el crecimiento promedio, calculado sobre el acumulado, varió entre $0,28 \mathrm{~cm}$ y $0,61 \mathrm{~cm}$, sugiriendo amplia variabilidad intraespecifica. 


\section{Cuadro $\mathrm{N}^{\circ} 3$ \\ PREDICCIÓN DE EFECTO ALEATORIO PARA CADA ÁRBOL Y SU IMPACTO SOBRE PREDICCIONES DE TASAS DE CRECIMIENTO PROMEDIO}

\begin{tabular}{|c|r|c|c|}
\hline Efecto aleatorio & Predicción & $\begin{array}{c}\text { Predicción a } \\
\text { los 55 años }\end{array}$ & $\begin{array}{c}\text { Predicción a } \\
\text { los } \mathbf{8 5} \text { años }\end{array}$ \\
\hline $\mathbf{u}_{1}$ & 5,9597 & 0,49 & 0,44 \\
\hline $\mathbf{u}_{2}$ & 14,9927 & 0,54 & 0,49 \\
\hline $\mathbf{u}_{3}$ & $-17,9082$ & 0,35 & 0,32 \\
\hline $\mathbf{u}_{\mathbf{4}}$ & $-16,1182$ & 0,36 & 0,33 \\
\hline $\mathbf{u}_{5}$ & 1,7970 & 0,47 & 0,42 \\
\hline $\mathbf{u}_{6}$ & 37,8321 & 0,67 & 0,61 \\
\hline $\mathbf{u}_{7}$ & 17,5557 & 0,57 & 0,50 \\
\hline $\mathbf{u}_{8}$ & $-17,8341$ & 0,35 & 0,32 \\
\hline $\mathbf{u}_{9}$ & $-25,8513$ & 0,31 & 0,28 \\
\hline $\mathbf{u}_{10}$ & $-0,4211$ & 0,45 & 0,41 \\
\hline
\end{tabular}

EI EAM fue igual a 0,087. El gráfico de residuos estudentizados vs valores estimados, no mostró ningún patrón, sugiriendo un buen ajuste.

\section{DISCUSIÓN}

La familia de ecuaciones no lineales de crecimiento asintótico es ampliamente utilizada en las ciencias forestales para modelar el crecimiento en especies arbóreas (Alder, 1980). Estas funciones pueden utilizarse en su forma integrada (función de rendimiento) y/o en su forma diferencial (función de crecimiento). En montes naturales, o donde se espera alta variabilidad entre individuos de la misma especie respecto a la tasa de crecimiento, resulta apropiado estimar los parámetros de estas ecuaciones en el contexto de los modelos mixtos que presuponen alta variabilidad en los crecimientos asintóticos entre individuos.

Para algarrobo blanco en el Chaco Semiárido argentino estos modelos sugieren resultados promedios similares a los previamente reportados. La tasa de crecimiento promedio es de 0,40 cm/año con una disminución de la misma alrededor de los 60 años de vida del árbol. Según prácticas forestales recomendadas (Perpiñal et al., 2005) la edad de culminación biológica se da recién a los 90 años de edad. Juárez de Galindez et al. (2005) con este mismo modelo para la estructura de medias o valor esperado del crecimiento, pero considerando efectos fijos y errores independientes, encuentran que la disminución del crecimiento en algarrobo blanco se observa después de los 30 años. Los errores estándares relativos y el EAM del modelo logístico mixto fueron menores a los encontrados por los mismos autores para el modelo logístico de efectos fijos (Galindez et al., 2005). Gimenez et al. (1998) para esta misma especie sugiere una tasa de crecimiento radial promedio de $0,40 \mathrm{~cm} /$ año que es superior al encontrado por Gimenez et al. en el año 2000 para Prosopis nigra $(0,33 \mathrm{~cm})$. Navall y Senilliani (2004) encuentran que en plantaciones (con tratamientos de distinta densidad y raleos) de esta especie en la zona de riego del Rio Dulce (Santiago del Estero, Argentina), el incremento anual 
en promedio es igual a $2,1 \mathrm{~cm}$, por lo que se puede inferir que en bosques nativos el crecimiento se ve fuertemente afectado por la competencia con malezas y otros árboles.

\section{CONCLUSIONES}

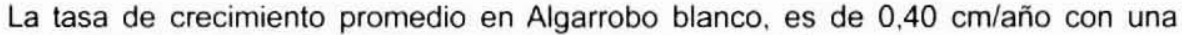
disminución de la misma alrededor de los 60 años de vida del árbol.

Para esta especie, la edad de culminación biológica se da recièn a los 90 años de edad.

\section{REFERENCIAS}

Alder, D. , 1980. Estimación del volumen forestal y predicción del rendimiento con referencia especial a los trópicos. Estudio FAO: Montes. Volumen 2. PP 56-61

Aniol, R., 1991. Computer aided tree rings analysis system, User manual. Schleswig. F.R.G. Alemania.

Balzarini, M., Macchiavelli, R, Casanoves, F., 2004. Curso-Taller Aplicaciones de Modelos Mixtos en Agricultura y Foresteria. CATIE Costa Rica.

Bolleta, P., 1988. Capitulo 1 de: Desmonte y habilitación de tierras en la región chaqueña semiárida Oficina regional de la FAO para América latina y El Caribe. Chile.

Catalàn, L., 2000. Crecimiento leñoso de Prosopis flexuosa en una sucesión post-agricola en el Chaco Árido efectos y relaciones de distintos factores de proximidad. Tesis doctoral. $230 \mathrm{p}$.

Cleveland, W.S., 1979. Robust locally weighted regression and smoothing scatterplots. Journal of American Statistical Association. 74:829-836.

Graybill, F. A., 1976. Theory and Application of the Linear Model. Wadsworth Publishing Company. 704 $\mathrm{pp}$.

Gimenez, A.M.; Moglia, J.G.; Hernández, P.; Bravo, S., 2000. Leño y la corteza de Prosopis nigra (Griseb.) Hieron, Mimosaceae, en relación a algunas magnitudes dendrométricas. Revista Forestal Venezolana. 44(2). 29-37

Giménez, A. M., 1998. Influencia de la Edad sobre caracteres anatómicos y el crecimiento de Schinopsis quebracho colorado Engl., Anacardiaceae. Tesis Doctoral.

Gimenez, Ana Maria; Ríos, Norfol; Moglia, Graciela y López, Carlos, 1998. Leño y corteza de Prosopis alba Griseb., algarrobo blanco, Mimosaceae, en relación con algunas magnitudes dendrométricas Revista Bosque 19(2):53-62

InfoStat, 2008. Infostat, versión 2008. Manual del usuario. Grupo Infostat, FCA, Universidad nacional de Córdoba. Primera Edición. Editorial Brujas. Argentina. 334 pp.

Juárez de Galindez, M.; Moglia, J.G.; Giménez A.M.; Pece, M. (2007)a. "Comparación de dos modelos de crecimiento de efectos fijos y errores independientes en Quebracho blanco". Revista Forestal 
Venezolana. 50-1. ISSN: 0556-6606.

Juárez de Galindez, M.; Gimenez, A.M.; Rios, N.; Balzarini, M. (2007)b. “Modelación del crecimiento en diámetro de vinal (Prosopis ruscifolia), en Santiago del Estero, Argentina". Revista Foresta Veracruzana No9. Volumen 2. ISSN 1405-7247.

Juárez de Galindez, M; Giménez, A. M.; Rios N.; Balzarini, M., 2005. Modelación de crecimiento en Prosopis alba Griseb. empleando dos modelos biológicos" Revista Quebracho N ${ }^{\circ} 12$. pag.34. ISSN 0328-0543

Juárez de Galindez, M.; Giménez, A.M.; Pece, M. y Rios, N., 2003. "Comparación de la aplicación de dos modelos de efectos fijos y errores independientes en el crecimiento de Schinopsis quebrachocolorado Engl."Revista Foresta Veracruzana 5(1)15-22. ISSN 1405-7247.

Juárez de Galindez, M.; Balzarini, M., 2003. "Modelación de la estructura de covarianza entre observaciones de un mismo individuo en modelos de curvas de crecimiento arbóreo". Revista de la SAE (on-line). V $17 \mathrm{~N}^{\circ} 1-2$. ISSN 0329-5583

Juárez de Galindez, M., 2001. Modelización estadistica de curvas de crecimiento de árboles en bosques nativos: quebracho colorado, quebracho blanco y algarrobo blanco. Tesis para optar al grado académico de Magister en Estadistica Aplicada. 131 pp.

Lee, Jack C., 1982. Classification of growth curves. In handbook de Statistics, Vol.2, 121-137. Krishnariah, P.R. and L.N.Kanab(Eds.) North Holland, Amsterdam.

Littell, R.; Milliken G.; Stroup, W. y Wolfinger, R., 1996. SAS System for Mixed Models. SAS Institute Inc., Cary, NC, USA. ISBN 1-55544-779-1.633 pp.

Lindstrom, M. y Bates, D., 1990. Nonlinear mixed effects models for repeated measures data. Biometrics 46, 673-687.

Machiavelli, R., 2007. Minicurso: "Aplicaciones de modelos no lineales mixtos". San Martin de los Andes, Argentina.

Moglia, J. G., 1999. Variabilidad de los caracteres anatómicos del leño de Aspidosperma quebracho blanco (Schecht), Apocinácea. Tesis doctoral. $91 \mathrm{p}$.

Molenberghs y Verbeke, 2007 Likelihood Ratio, Store and Wald Tests in a constrained Parameter Space. The American Statistician, February 2007, Vol.61, №1.

Naumann, M.; Madariaga, M. (2003).Atlas Argentino/Argentinienatlas. Programa de acción nacional de lucha contra la desertificación. Secretaria de Ambiente y Desarrollo Sustentable. Instituto Nacional de tecnologia Agropecuaria. Deutsche Gesellschaft für Technische Zusammenarbeit. 94 pp. ISBN: $987-$ 20598-5-3. Buenos Aires.

Navall, M. y Senilliani, M.G., 2004. Crecimiento y calidad maderera de plantaciones de Prosopis alba Griseb (Algarrobo blanco) en el área de riego del Rio Dulce, provincia de Santiago del Estero. XIX Jornadas Forestales de Entre Rios. Argentina.

Perpiñal, E.; M.Balzarini; L. Catalán; L. Pietrarelli y U. Karlin, 1995. Edad de culminación del crecimiento en Prosopis flexuosa D.C. en el Chaco Árido Argentino. Investigación Agraria. Sistemas y Recursos Forestales . 4(1) :45-55. 
Rios N.; A. M. Gimenez y J. G. Moglia de Lugones, 1997. Crecimiento de especies leñosas de la Región Chaqueña Seca. AFOA. Actas /I Congreso Forestal Argentino y Latinoamericano. ISSN: 03291103. Bosques Nativos y Protección Ambiental. $10 \mathrm{p}$.

Rios, N., Giménez, A. M. y Torales, A., 1996. Análisis Epidométrico de fuste. /l Jornadastécnicas del Chaco. Formosa. $18 \mathrm{p}$.

Villalba, R., 1988. Dendrocronología: su aplicación al manejo dasonómico de los algarrobales.

Wolfinger, R., 1993. Fitting Nonlinear Mixed Models with the New NLMIXED procedure. Paper 287. SAS Institute Inc., Cary, NC. 